\title{
Knowledge, Attitudes and Practices Survey in Management of Type 2 Diabetes by General Practitioners in Dakar
}

\author{
Abdoulaye Leye*, Nafy Ndiaye, Ngoné Diaba Diack, Michel Assane Ndour, Biram Codou Fall, \\ Yakham Mohamed Leye, Papa Ousseynou Mane
}

Internal Medicine and Endocrinology Department, Teaching Hospital of Pikine, Dakar, Senegal

Email: *ablayleye@hotmail.com

How to cite this paper: Leye, A., Ndiaye, N., Diack, N.D., Ndour, M.A., Fall, B.C., Leye, Y.M. and Mane, P.O. (2017) Knowledge, Attitudes and Practices Survey in Management of Type 2 Diabetes by General Practitioners in Dakar. Journal of Diabetes Mellitus, 7, 294-301.

https://doi.org/10.4236/jdm.2017.74024

Received: October 8, 2017

Accepted: November 5, 2017

Published: November 9, 2017

Copyright $\odot 2017$ by authors and Scientific Research Publishing Inc. This work is licensed under the Creative Commons Attribution International License (CC BY 4.0).

http://creativecommons.org/licenses/by/4.0/

\begin{abstract}
Introduction: Considering the global burden of diabetes and lack of specialist in diabetology in our subsaharian area, general practitioners (GPs) play a major role as first referent for care of diabetic people. The aim of this work was to describe knowledge, attitudes and practices of GPs related to management of type 2 diabetes. Patients and Methods: It was about a transversal and descriptive survey held between July 14, 2015 and November 1st, 2015 in the medical districts and public corporations of health of Dakar in Senegal. Results: The population of study was made of 107 males and 40 females GPs. The majority $(82.8 \%)$ was graduated since less than five years. Hygienic and dietetic measures (HDM) were known and commonly recommended by $77.60 \%$ of GPs in their daily practice. The caloric intakes were known by $21.1 \%$. The 30 min of physical activity per day three times in the week was advised by $91.20 \%$. Monotherapy with metformine associated with HDM was prescribed by $76.90 \%$. Bitherapy with metformine and sulfonylureas associated to HDM were prescribed by $39.50 \%$. Concerning insulin therapy, mixed insulin twice daily associated with rapid insulin before lunch was prescribed by $49.7 \%$. Use of rapid acting insulin with three injections before each meal was prescribed by $36.10 \%$. Treatment of the other cardiovascular risk factors was addressed by $97.30 \%$. Smoking cessation was advised by $37.40 \%$. Use of sweetened drink and intravenous glucose were most adopted in case of hypoglycemia, and use of glucagon was adopted by $23.10 \%$. To improve the diabetic retinopathy $61.2 \%$ of GPs recommended tight control of other cardiovascular risk factors. Rigorous glycemic control and regular physical activity were recommended for painful neuropathy beside analgesics. Smoking cessation was also recommended by $61.90 \%$ for that. Against obstructive arterial disease of the lower extremities, tight control of blood pressure was recom-
\end{abstract}


mended by $69.40 \%$ Conclusion: It comes out from these results the need for reinforcing post graduate trainings on diabetes for our GPs. Moreover, there's an urgent need to elaborate and disseminate adapted guidelines and recommendations for improving efficient and standardized strategies for day to day management of type 2 diabetic people in our country.

\section{Keywords}

Knowledge, Attitudes, Practices, Diabetes, General Practitioners

\section{Introduction}

The dramatic increase in diabetes has occurred in all countries, and in rural as well as urban areas. According to IDF projections [1], an estimate of 415 million cases (uncertainty interval: 340 - 536 millions) of diabetes among adults aged 20 - 79 years in 220 countries and territories for 2015 was established. For 2040, it was estimated that 642 million (uncertainty interval: 521 - 829 millions) people aged 20 - 79 will have diabetes. Diabetes research includes trying to find a cure for diabetes, improving diabetes medication and diagnostics, and making the day to day life of people with diabetes easier to lead.

The epidemiological studies conducted in Africa show that the diagnosis of diabetes is often ignored ( 2 to 3 cases not diagnosed for each known case). This situation had been pointed out since first publications on diabetes in Africa [2]. In most case these patients present themselves with one of the complications of the diabetes already set up as shown in Senegal [3], where there is less than 10 specialists in diabetology for 14 million inhabitants. So, the general practitioners are the major actors in the global strategy for caring diabetic patients [4]. The aim of this survey was to evaluate knowledge, attitudes and practices of the general doctors on management of type 2 diabetes with specific focus on managing hyperglycemia, other cardiovascular risk factors and degenerative complications prevention.

\section{Methodology}

\subsection{Investigation}

It was an observational survey, transversal and descriptive nearby GPs of the medical districts and public establishments of health of the area of Dakar. After having presented the aims of the study to the persons in charge of structures in order to obtain their authorization of investigation, a questionnaire was selfmanaged with each GP. The investigation was constituted by a questionnaire form including questions with multiple choices. The questionnaire consisted of 2 parts, a first part on the socio-professional characteristics of the GPs (sex, seniority, place of exercise, previous training on diabetes) and a second part related to knowledges, attitudes and practices in managing of hyperglycemia, de- 
generative complications as well as the associated cardiovascular risk factors.

\subsection{Statistical Analysis}

The seizure and the data analysis were carried with the software EPI Info version 3.3.2. This software allowed to calculate the frequencies, the means and the standard deviations.

\section{Results}

\subsection{Socio-Professional Characteristics}

On 170 questionnaires delivered, 152 had been recovered. Only 147 questionnaires were exploitable. The population of study consisted of 107 men (72.80\%) and 40 women (27.20\%). According to their seniority of exercise, $82.8 \%$ were graduated since less than 5 years. Eighty GPs (54.4\%) had received a continuous medical training on diabetes.

\subsection{Knowledge of the Non Insulinic Treatments of Type 2 Diabetes}

The main categories of antidiabetic medicines were known, however there were confusions between molecules, their side-effects and their counter-indications. Metformin was regarded as sulfonylurea by $28.60 \%$ of respondents. Respectively 20 (13.6\%), 16 (10.8\%) and 14 (9.5\%) GPs considered Glibenclamide, Gliclazide and Glipizide like biguanides. Only 1 GP had recognized Glipizide as part of the sulfonylureas. Hypoglycemia was regarded as side-effect of the biguanides by $44.90 \%$ of GPs and for $35.5 \%$ of GPs, the sulfonylureas were providers of digestive disorders. Table 1 summarizes the distribution of the GPs according to their

Table 1. Distribution of the GPs according to their knowledge of the non insulinic treatments of type 2 diabetes.

\begin{tabular}{|c|c|c|}
\hline & Number & Percentage \\
\hline \multicolumn{3}{|l|}{ Classes of non insulinic drugs } \\
\hline Biguanides & 142 & $96.6 \%$ \\
\hline Sulfonylureas & 146 & $99.3 \%$ \\
\hline Incretin mimetics & 7 & $7.76 \%$ \\
\hline Alpha-glucosidase Inhibitors & 96 & $65.3 \%$ \\
\hline Glinides & 118 & $80.30 \%$ \\
\hline \multicolumn{3}{|l|}{ Main side effect of biguanides } \\
\hline Metformin associated lactic acidosis & 66 & $44.9 \%$ \\
\hline \multicolumn{3}{|l|}{ Main side effect of sulfonylureas } \\
\hline Hypoglycemia & 140 & $95.2 \%$ \\
\hline \multicolumn{3}{|l|}{ Contraindications of sulfonylureas } \\
\hline Pregnancy & 91 & $61.90 \%$ \\
\hline Liver failure & 81 & $55.1 \%$ \\
\hline \multicolumn{3}{|l|}{ Contraindications of biguanides } \\
\hline Kidney failure & 89 & $60.5 \%$ \\
\hline
\end{tabular}


knowledge of the non insulinic drugs.

\subsection{Practices of the GPs for Management of Hyperglycemia for Type 2 Diabetic Patients}

One hundred fourteen GPs (77.6\%) recommended the HDM during all treatment long. Seventy-five (51.0\%) found that HDM could be enough to normalize glycaemia for a long time, and 58 GPs $(39.5 \%)$ considered it as first line treatment.

For initial monotherapy prescription, $34.7 \%$ of GPs recommended to start metformin after failure of HDM and $76.9 \%$ considered the possibility to start Metformin at diagnosis at the same time with HDM. Use of sulphonylureas as possible monotherapy after failure of HDM was suggested by $11.6 \%$ of GPs and while for $19.0 \%$ of them, it was suggested as first line monotherapy at diagnosis at the same time with HDM. For $39.5 \%$ of GPs, the most recommended bitherapy was association of metformin plus suphonylureas.

\subsection{Practices of Insulin Therapy in Type 2 Diabetes by GPs}

Insulin was recommended when failure of non insulinic therapeutics was proven $(87.80 \%)$ and in case of severe intercurrent infections (76.90\%). Fifty-six doctors (38.10\%) prescribed insulin when HbAlc was higher or equal to $9 \%$ and 55 among them (37.4\%) prescribed it when the fasting blood glucose was higher than $3 \mathrm{~g} / \mathrm{L}$.

After failure of oral hypoglycemic agent, premixed insulin with $2 / 3$ of total dose administrated in the morning and $1 / 3$ in the evening associated with a fast acting insulin before lunch, was adopted by $49.7 \%$ of GPs.

For 53 GPs (36.10\%), the regimen of insulin therapy suggested was rapid acting insulin three time per day, one injection before each meal then.

\subsection{Knowledges and Practices of GPs in Management of the Degenerative Complications of Type 2 Diabetes}

\section{- Diabetic retinopathy}

One hundred thirty $(88.40 \%)$ and 98 (66.70\%) GPs had respectively recognized the maintenance of optimal glycemic balance and the maintenance of a stable blood pressure balance as factors to prevent diabetic retinopathy. Thirtysix doctors $(24.50 \%)$ were aware of possibility of photocoagulation with laser. One hundred thirty-five $(91.80 \%)$ had recognized that fundus examination of eye as soon as type 2 diabetes is diagnosed and then each year, as well the most optimal balance of glycaemia could allow an early tracking and the prevention of the diabetic retinopathy. Ninety GPs (61.20\%) had considered that good control of cardiovascular risk factors could also allow a prevention of diabetic retinopathy retinopathy.

\section{- Diabetic neuropathy}

One hundred eight GPs (73.50\%) had adopted the antalgic treatment in case of painful neuropathy. Only $47(32.00 \%)$ and 27 (18.40\%) GPs respectively 
adopted the treatment by nerve sedatives and insulin therapy in case of painful peripheral neuropathy. For the prevention of neuropathy, 138 (93.90\%) and 113 (76.90\%) had recognized respectively that rigorous glycemic control and regular physical activity could allow a prevention of diabetic neuropathy. Smoking cessation also was recommended by 91 (61.90\%) GPs for this last objective.

\section{- Diabetic kidney disease}

Concerning the measures adopted in the event of nephropathy, the maintenance of an optimal glycemic balance and the maintenance of a perfect blood pressure balance were recognized respectively by 135 (91.80\%) and 102 (69.40\%) GPs. For the maintenance of an optimal blood pressure control, the use of Angiotensine converting enzyme inhibitors (ACE inhibitors) was more frequently adopted $(49.00 \%$ of GPs) than angiotensin receptor blockers (ARBs) $(34.70 \%$ of GPs).

One hundred thirty-three GPs (90.50\%) and 123 (83.70\%) recognized respectively that the strict control of glycaemia and blood pressure could help to prevent diabetic nephropathy. The reduction of food protein intake ration as a way to prevent diabetic nephropathy, was recommended by 44 (13.60\%) GPs.

\section{- Peripheral artery disease}

For the drugs used in case of obliterating arteriopathy of the lower limb, antiplatelet agents were mostly prescribed ( $81.60 \%$ of GPs) followed by arterial vasodilators $(52.40 \%$ of GPs) and statins ( $46.90 \%$ of GPs). Obtaining a good glycemic control and the correction of a dyslipidemia were recommended by respectively $92.50 \%$, and $81.60 \%$ of GPs for better management of peripheral artery disease. Tight control of glycaemia and blood pressure was also recommended by $69.40 \%$ of GPs.

\section{- The diabetic foot}

One hundred twenty-nine (87.80\%) GPs and 122 (83.00\%) respectively adopted optimized insulin therapy and antibiotic therapy by general route. Prevention with tetanus vaccine was recommended by $80.30 \%$ of GPs. For the prevention of the diabetic foot, the daily hygiene of feet and the adequate foot wearing were applied by respectively 140 (95.20\%) and 139 (94.60\%) of GPs. The control of glycemic balance was also recognized by 136 (92.50\%) GPs.

Other preventive measures had been recommended by $2.7 \%$ of GPs (self-inspection of feet, avoiding the exposure to sources of heat, never tearing off a scale, avoiding foot lesions).

\subsection{Practices of GPs in Management of Other Cardiovascular Risk Factors}

\section{- Hypertension}

In first intention, 98 GPs (66.70\%) adopted the treatment by ACE inhibitors of the and 18 GPs (12.20\%) proposed associations of antihypertensive drugs. In second intention, 49 GPs (33.30\%) proposed the use of ARBs.

\section{- Dyslipidemia}

The majority of GPs (86.40\%) recommended statins and $85.70 \%$ of them in- 
dicated diet against dyslipidemia. The maintenance of good glycemic balance was advocated by $69 \mathrm{GPs}(46.9 \%)$.

\section{- Obesity}

One hundred thirty-six GPs (92.50\%) and 101 (68.70\%) respectively advised regular physical-activity and hypocaloric diet for their obese diabetic patients. Use of hypolipidemic drugs was advised that by 44 GPs (29.90\%) for this situation of obesity

\section{- Smoking}

One Hundred thirty GPs (89.10\%) advised immediate smoking cessation and dedicated tobaccologic consultation was advised by 55 (37.40\%) GPs.

\section{Discussion}

Epidemiological studies made in our African areas emphasize on the significant role of the GPs in the global strategy of managing type 2 diabetic patients because of lake of medical doctors in general, and diabetologist in particular [4]. However, that should rest on clear and adapted therapeutic recommendations, taking into account the associated cardiovascular risk factors. It passes by improvement of initial university and post-graduate trainings as demonstrated in Algeria before [5]. Only one half of our GPs had already received a specific postgraduate training on type 2 diabetes management.

The place of HDM in type 2 diabetes management is overall well-known by our GPs as in previous similar study in Senegal [6]. Among them, 39.5\% put forward the HDM associated with Metformin as first step of the treatment as recommended by ADA/EASD position statement [7]. Monotherapy with sulphonylureas was seldom prescribed (19.00\%). AMAR-AFO Study found that sulphonylureas were prescribed in $67.8 \%$ of monotherapy, including $54.5 \%$ for glimepiride and $13.3 \%$ for gliclazide [4]. Only $7.76 \%$ of the GPs knew the existence of the incretin mimetics. This lack of knowledge could be explained by the absence of continuous medical training leading to lack of update about new therapeutics. Bitherapy combining sulphonylureas and metformine associated with HDM was prescribed by $39.50 \%$ GPs. This rate was higher the one found in DiabCare Senegal study [6] in which bitherapy was prescribed only in $10.1 \%$ of the cases in dedicated centers to diabetes care. No other combination of bitherapy was proposed in our studies. It could be justified by lack of availability and/or affordability of other hypoglycemic classes (including incretin mimetics, glinides and glitazone) in our country. As well, fears and barriers for insulin therapy doesn't promote bitherapy with metformin and insulin, comparatively to oral hypoglycemic agents' combination, as recently recommended in international guidelines comparatively to previous ones [7] [8] [9].

The majority of GPs prescribed insulin in the event of proven failure of oral therapeutic (87.80\%) and severe intercurrent infections (76.90\%).

For all type 2 diabetic people, a comprehensive approach with multiple facets must be adopted to reduce the cardiovascular risk. Methods for vascular protec- 
tion include lifestyle modification (food mode, weight loss, rise of the physical activity, weaning of the tobacco) and pharmacological treatments (antiplatelet agents, statins, ACE inhibitors, ARBs, control of glycaemia and blood pressure) [7]. The management of these other cardiovascular risk factors was overall well known by more than $85 \%$ of GPs, despite some misconceptions. An efficient use of international guidelines requires minimal adaptation to our environment and resources to make it comprehensive as needed in others developing countries [10].

The broad outlines for management of degenerative complications were known. However, there was some mix-up like recommendation by $23.10 \%$ of GPs for the use of glucagon in the event of hypoglycemia occurring in type 2 diabetes without consideration of the etiology. This fact, as example of misunderstanding among others underline the urgent need for implementation of continuous medical education programs whose benefit has been demonstrated before elsewhere [11].

\section{References}

[1] Ogurtsova, K., Da Rocha Fernandes, J.D., Huang, Y., Linnenkamp, U., Guariguata, L., Cho, N.H., Cavan, D., Shaw, J.E. and Makaroff, L.E. (2017) IDF Diabetes Atlas: Global Estimates for the Prevalence of Diabetes for 2015 and 2040. Diabetes Research and Clinical Practice, 128, 40-50. https://doi.org/10.1016/j.diabres.2017.03.024

[2] Payet, M., Sankale, M., Pene, P., et al. (1960) The Chief Aspects of Diabetes Mellitus in an African Environment at Dakar. Bull Soc Pathol Exot Filiales, 53, 901-910.

[3] Mbaye, M.N., Niang, K., Sarr, A., et al. (2011) Epidemiologic Aspects of Diabetes in Senegal. Medecine des Maladies Métaboliques, 5, 659-664.

[4] Diop, S.N., Wade, A., Lokrou, A., Diedhiou, D. and Adoueni, V.K. (2013) Management of Type 2 Diabetes in Clinical Practices in Sub-Saharan Africa: Results of the AMAR-AFO Study in Senegal and Ivory Cost. Medecine des maladies métaboliques, 7, 363-367.

[5] Malek, R., Roula, D., Belhadj, M.R., et al. (2015) Medical Continues Training on Diabetes for General Practitioners in Algeria, 2004-2014. Médecine des maladies Métaboliques, 9.

[6] Mbaye, M.N., Sarr, A., Diop, S.N., et al. (2011) DiabCare Senegal: An Investigation on the Management of Diabetes in Senegal. Medecine des Maladies Metaboliques, 5, 85-89.

[7] Inzucchi, S.E., et al. (2015) Management of Hyperglycemia in Type 2 Diabetes, 2015: A Patient-Centered Approach: Update to a Position Statement of the American Diabetes Association and the European Association for the Study of Diabetes. Diabetes Care, 38, 140-149. https://doi.org/10.2337/dc14-2441

[8] Halimi, S., Grimaldi, A., Gerson, M., et al. (2011) Medicinal Treatment of Type 2 Diabetic Patient. The New Recommendations. http://www.afssaps.fr

[9] Inzucchi, S., Bergenstal, R., Buse, J., et al. (2012) Management of Hyperglycemia in Type 2 Diabetes: A Patient-Centered Approach. Position Statement of the American Diabetes Association (ADA) and the European Association for the Study of Diabetes (EASD). Diabetes Care, 35, 1364-1379. https://doi.org/10.2337/dc12-0413 
[10] Widyahening, I.S., Wangge, G., Van der Graaf, Y. and Van der Heijden, G.J. (2017) Adapting Clinical Guidelines in Low-Resources Countries: A Study on the Guideline on the Management and Prevention of Type 2 Diabetes Mellitus in Indonesia. Journal of Evaluation in Clinical Practice, 23, 121-127. https://doi.org/10.1111/jep.12628

[11] Aghili, R., Malek, M., Baradaran, H.R., Peyvandi, A.A., Ebrahim Valojerdi, A. and Khamseh, M.E. (2015) General Practitioners' Knowledge and Clinical Practice in Management of People with Type 2 Diabetes in Iran; The Impact of Continuous Medical Education Programs. Archives of Iranian Medicine, 18, 582-585. 